\title{
Pathophysiology of Myocardial Injury and Remodeling: Implications for Molecular Imaging
}

\author{
Jennifer A. Dixon and Francis G. Spinale
}

Division of Cardiothoracic Surgery, Medical University of South Carolina, and Ralph H. Johnson VA Medical Center, Charleston, South Carolina

\begin{abstract}
Despite advances in reperfusion therapy, acute coronary syndromes can still result in myocardial injury and subsequent myocardial infarction (MI). Molecular, cellular, and interstitial events antecedent to the acute $\mathrm{MI}$ culminate in deleterious changes in the size, shape, and function of the left ventricle (LV), collectively termed LV remodeling. Three distinct anatomic and physiologic $L V$ regions can be described after Ml: the infarct, border zone, and remote regions. Given the complexity of post-MI remodeling, imaging modalities must be equally diverse to elucidate this process. The focus of this review will first be on cardiovascular MRI of the anatomic and pathophysiologic LV regions of greatest interest with regard to the natural history of the post-MI remodeling process. This review will then examine imaging modalities that provide translational and molecular insight into burgeoning treatment fields for the attenuation of post-MI remodeling, such as cardiac restraint devices and stem cell therapy.
\end{abstract}

Key Words: basic cardiology; technical cardiology; clinical cardiology; MRI; cardiac MRI; infarct expansion; myocardial remodeling

J Nucl Med 2010; 51:102S-106S

DOI: 10.2967/jnumed.109.068213

\section{A} cute coronary syndrome, which can be defined as a constellation of clinical symptoms associated with acute myocardial ischemia, strikes nearly a million people in the United States each year ( 1$)$ and is responsible for tens of billions of dollars in hospital charges (2). Optimal treatment with timely reperfusion therapy and pharmacologic intervention has made acute coronary syndrome increasingly survivable, even in the face of a rising incidence given the growing elderly population (1). Despite advances in reperfusion therapy, acute coronary syndromes can still result in myocardial injury and subsequent myocardial infarction (MI), evoking cellular and extracellular processes in the reperfusion phase leading to cell death, inflammation, and scar formation. Molecular, cellular, and interstitial events antecedent to the acute MI culminate in changes in

Received Sep. 1, 2009; revision accepted Jan. 15, 2010.

For correspondence or reprints contact: Francis G. Spinale, Cardiothoracic Surgery, Strom Thurmond Research Center, 114 Doughty

St., Suite 625, Charleston, SC 29425.

E-mail: wilburnm@musc.edu

COPYRIGHT @ 2010 by the Society of Nuclear Medicine, Inc. the size, shape, and function of the left ventricle (LV), collectively termed LV remodeling. Despite successful reperfusion, lifestyle modification, and pharmacotherapy, the LV remodeling process continues unabated, resulting in an accrual rate of almost 1 million new patients per year at risk for developing heart failure (1). Accordingly, strategies to selectively and specifically monitor the molecular pathways that underlie the LV remodeling process hold great import to alleviate the socioeconomic and healthcare resource burden of post-MI remodeling.

To identify appropriate imaging targets in the context of post-MI LV remodeling, the anatomic and biologic underpinnings of the process must be considered. For the purposes of the remodeling process, the post-MI LV can be divided into 3 distinct anatomic regions: the MI itself, the surrounding border zone, and the remaining remote myocardium. Regardless of reperfusion, degradation of normal extracellular matrix occurs within the MI region, accompanied by invasion of inflammatory cells and induction of bioactive peptides and cytokines, leading to the necessary substructure for scar formation $(3,4)$. The fully perfused myocardium surrounding the MI, described as the border zone, is a metabolically active amalgam of inflammatory cells, fibroblasts, and viable myocytes. The border zone is also the site of infarct expansion, a dynamic process defined as the extension of changes in structure and function between the MI and remote myocardium. Infarct expansion is a process of great import, as it has been identified as an independent predictor of mortality for post-MI patients (5). The remote region is normally perfused and can be millimeters to centimeters away from the MI region and border zone. Despite conventional belief, the remote region is not immune to the post-MI remodeling process, as changing wall strain patterns after MI lead to myocyte hypertrophy and interstitial fibrosis (6). Each of these 3 anatomic regions is distinctly different, and therefore the heterogeneity of the regions and cellular targets within the post-MI LV must be recognized in order to achieve appropriate imaging modalities.

Although post-MI remodeling is a continually evolving process, the 30th day after MI can be described as a milestone time point at which the infarct itself is roughly healed. 


\begin{tabular}{|c|c|c|}
\hline Domain & Target & Reference \\
\hline \multirow{3}{*}{$\begin{array}{l}\text { Extracellular } \\
\text { matrix }\end{array}$} & Collagen & 7,8 \\
\hline & Matrix metalloproteinases & 14,15 \\
\hline & $\begin{array}{l}\text { Tissue inhibitors of matrix } \\
\text { metalloproteinases }\end{array}$ & 14,15 \\
\hline \multirow[t]{3}{*}{ Vasculature } & Integrins & 13 \\
\hline & $\begin{array}{l}\text { Vascular endothelial } \\
\text { growth factor }\end{array}$ & $10,11,12$ \\
\hline & Smooth muscle actin & 17 \\
\hline \multirow[t]{3}{*}{$\begin{array}{l}\text { Signaling/growth/ } \\
\text { viability }\end{array}$} & $\begin{array}{l}\text { Transforming growth } \\
\text { factor- } \beta\end{array}$ & 18 \\
\hline & Apoptotic markers & 16 \\
\hline & Myofibroblasts & 9 \\
\hline
\end{tabular}

Several large-animal studies have been conducted examining this period of the remodeling process and the unique events happening in each of the 3 anatomic regions (7-18). Jugdutt et al. used a canine model to characterize the collagen dynamics, as well as the effects of angiotensinconverting enzyme inhibition on collagen deposition, within the infarct zone during this time frame $(7,8)$. Subsequently, MI collagen and the myofibroblasts that produce it have become molecular imaging targets, capable of reflecting the therapeutic effects of angiotensin-converting enzyme inhibition (9). In the border zone, vascular endothelial growth factor, a marker of angiogenesis during the post-MI remodeling process, has been studied in pigs, providing a molecular imaging target to assess border zone myocardial perfusion status (10-12). Integrins specific to angiogenic vessels have also been used as a novel target for imaging angiogenesis in post-MI large-animal models (13). Noninvasive imaging of angiogenesis in the border zone holds important implications for risk stratification of patients after MI and the potential to evaluate the response to therapeutic measures aimed at myocardial angiogenesis. Unique portfolios of matrix metalloproteinases, a family of proteases that have been shown to be integral to the extracellular matrix remodeling process, have been characterized in all post-MI regions in sheep models $(14,15)$ and provide noninvasive molecular imaging targets to interrogate the post-MI remod- eling process. Apoptotic markers are clearly involved with pathways that can contribute to adverse remodeling after MI (16). At present, the imaging of apoptotic pathways has been limited to small-animal models (19) but clearly holds relevance for clinically relevant large-animal models. A summary of domains of the biologic remodeling process (i.e., extracellular matrix, vasculature, and signaling pathways) is presented in Table 1, as well as potential imaging targets. Imaging of the post-MI remodeling process holds great import for both prognostic information and potential monitoring of therapeutic interventions.

Given the complexity of post-MI remodeling, imaging modalities must be equally diverse to elucidate this process. Consequently, it is not possible to integrate the entire body of imaging studies that are applicable to LV remodeling in this brief review. Rather, the focus of this review will first be on cardiovascular MRI of the anatomic and pathophysiologic LV regions of greatest interest with regard to the natural history of the post-MI remodeling process. Second, this review will examine imaging modalities that provide translational and molecular insight into burgeoning treatment fields for the attenuation of post-MI remodeling, such as cardiac restraint devices and stem cell therapy.

\section{POST-MI LV REMODELING: BIOPHYSICAL CHARACTERIZATION BY MRI}

By being able to provide accurate, reproducible images of high spatial and temporal resolution in any plane, cardiac MRI is especially suited for longitudinal assessment of post-MI LV remodeling (Fig. 1) (20,21). Several studies have used MRI techniques to gain molecular and cellular insight into the postMI remodeling process. Specifically, Friedrich et al. have demonstrated the use of MRI to characterize reversibly and irreversibly injured myocardium in and around the MI region (Fig. 2) (22). They hypothesize that mapping areas of post-MI myocardial edema, using T2-weighted and late enhancement MRI, can guide therapeutic modalities to the border zone in attempts to attenuate infarct expansion. Other reports have targeted the reduction of post-MI edema, as detected by MRI, as a novel method to attenuate $\mathrm{LV}$ remodeling at the molecular level (23). After MI, contrast-enhanced MRI techniques provide visualization of microvascular obstruction in the
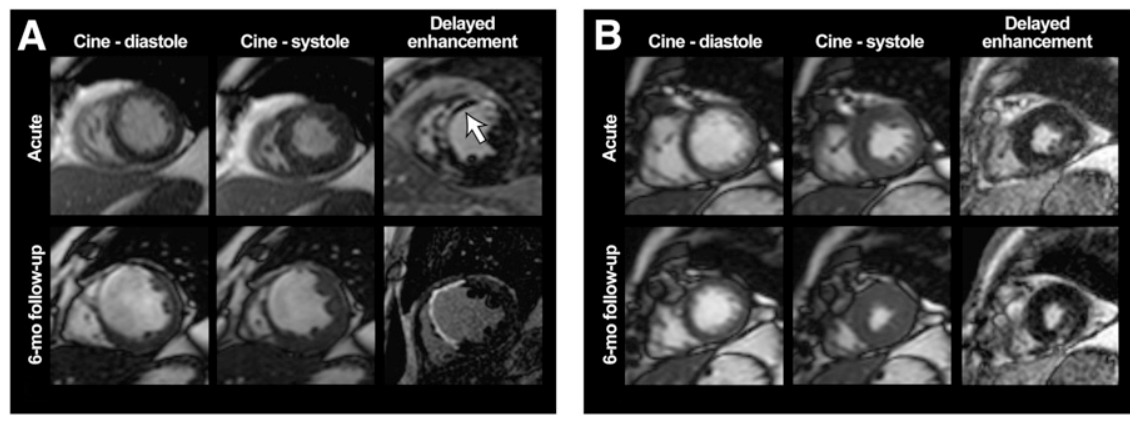

FIGURE 1. Examples of delayed-enhancement MRI of 2 different MIs demonstrating relationship between LV remodeling and functional recovery. (A) Large anteroseptal transmural acute $\mathrm{MI}$ with areas of microvascular obstruction within infarct region (arrow) is shown in acute phase. Images 6 mo after MI show substantial LV remodeling, with wall thinning and absence of regional functional recovery. (B) Small inferolateral subendocardial $\mathrm{Ml}$ with regional hypokinesis is shown in acute phase. Subsequent 6-mo post-MI images show lack of LV remodeling and complete recovery of regional contractility, in contrast to $\mathrm{MI}$ shown in $\mathrm{A}$. (Reprinted with permission of (21).) 

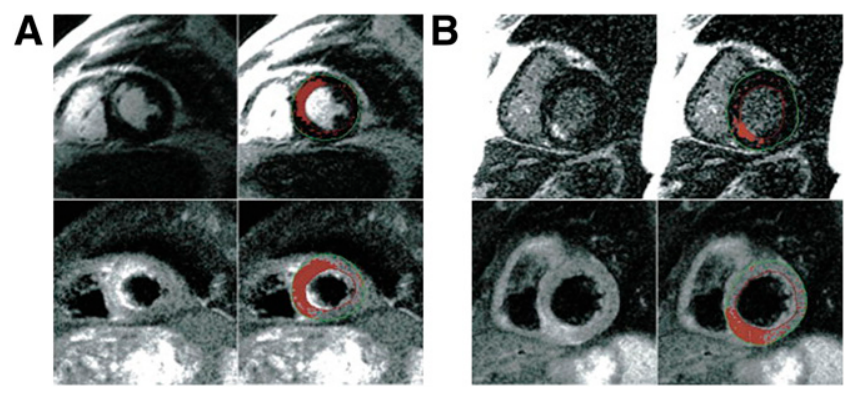

FIGURE 2. In 2 patients, irreversible and reversible injury in acute reperfused infarcts as visually and semiautomatically defined. (A) Cardiac MRI 3 d after acutely reperfused infarct with subtotal (99\%) occlusion of left anterior descending artery in 71-y-old patient. (B) Cardiac MRI $1 \mathrm{~d}$ after reperfusion of occluded right coronary artery in 57-y-old patient. Red areas indicate result of semiautomatic delineation of pixels with abnormal signal as defined by signal intensity more than 2 SDs above mean signal intensity of remote myocardium. Spatial extent of myocardial injury in edema-sensitive T2 imaging is consistently larger than that of necrosis-sensitive late enhancement. (Reprinted with permission of (22).)

infarct region (24). Regions of microvascular obstruction, which can persist despite reperfusion, appear as subendocardial dark areas surrounded by hyperenhanced injured myocardium and have been reported as a marker for adverse LV remodeling (25). In a landmark report by Wu et al., contrastenhanced MRI showed that microvascular obstruction and infarct size correlated with long-term prognosis, including the development of heart failure (26).

The topography of the LV after MI is a heterogeneous landscape, with great prognostic value held in the infarct characteristics and myocardial perfusion status $(27,28)$. This landscape is made traversable to researchers and clinicians with the help of a variety of imaging modalities. Although CT can provide high-resolution static images to identify the region of MI, the current consensus is that MRI is able to provide better cardiac images than CT $(29,30)$ with an additional feature of superior tissue characterization. For example, both clinical and preclinical studies $(31,32)$ have used diffusion tensor MRI to study myocardial fiber architecture characteristics, describing distinct unique qualities among the MI region, border zone, and remote region. Specifically, the percentage of left-handed helical fibers was shown to have increased from the remote zone $(13.3 \% \pm$ $5.8 \%)$ to the border zone $(19.2 \% \pm 9.7 \%)$ and infarct zone $(25.8 \% \pm 18.4 \%)$ in patients at a median of $26 \mathrm{~d}$ after MI (32). Myocardial fiber architecture correlated with infarct size and LV function in these studies, linking structural and functional post-MI remodeling (32). Furthermore, by identifying these distinct anatomic LV regions, this imaging technology can enable scientists and clinicians to intervene in the post-MI remodeling process.

Another technique that has been used to characterize the distinct myocardial regions involved in post-MI remodeling is magnetic resonance spectroscopy (MRS). A multitude of
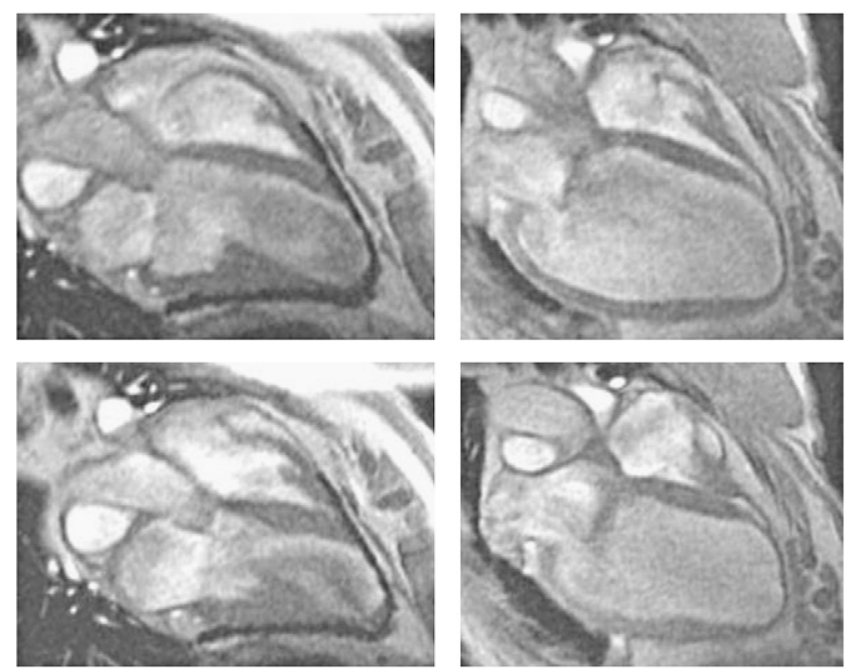

FIGURE 3. Long-axis MR images obtained $6 \mathrm{wk}$ after infarction of device (left) and control (right) sheep hearts. Hearts are shown in diastole in top panels and systole on bottom. Mesh appears as dark area around ventricles. (Reprinted with permission of (35).)

myocardial metabolites can be noninvasively detected using MRS, characterizing the regional metabolic changes that occur in post-MI remodeling. $\mathrm{Hu}$ et al. reported a $50 \%$ reduction in the phosphocreatine-to-adenosine triphosphate ratio in the border zone, as compared with the remote region of a porcine MI model, indicating that border-zone energy defects likely contribute to local dysfunction after MI (33). Feygin et al. used MRS to examine border-zone bioenergetics after mesenchymal stem cell injection, reporting significant improvement in the phosphocreatine-to-adenosine triphosphate ratio 4 wk after MI in a porcine model (34). Hence, MRS allows interrogation of specific myocardial regions after MI and provides an assessment of therapeutic response. Presently, however, the spatial and temporal resolution limitations of MRS have not yet allowed high-resolution cardiac metabolic imaging to become a clinical reality.

FIGURE 4. Epicardial elastic support device in dilated cardiomyopathy. Left panel shows preoperative cardiac MR images from patient with dilated cardiomyopathy. Right panel shows same patient 6 mo after placement of HeartNet (Paracor Medical, Inc.). (Courtesy of Robert W.W. Biederman, Allegheny General Hospital, Pittsburgh, PA).
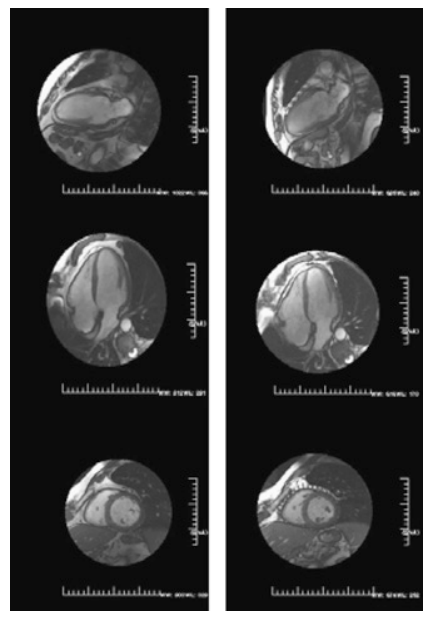
FIGURE 5. In vivo serial MRI. Serial short-axis views obtained at 1 (A), 2 (B), 4 (C), and 10 (D) wk show persistent signal void after injection of mesenchymal stem cells labeled with ferumoxides (Feridex; Bayer HealthCare Pharmaceuticals). (Reprinted with permission of (37).)
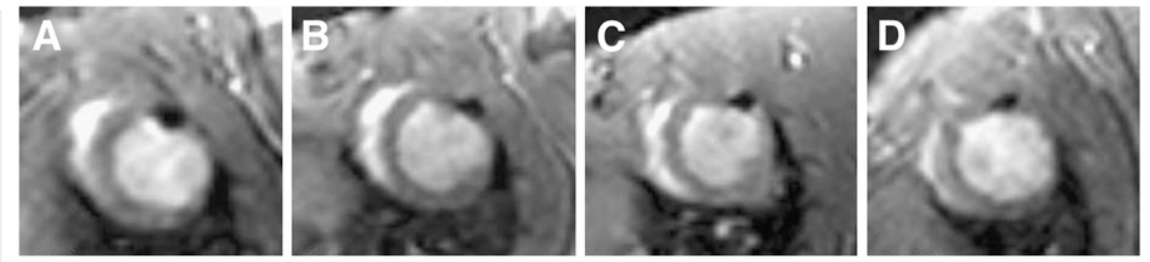

\section{REGIONAL IMAGING AFTER MI: PROGNOSTIC AND THERAPEUTIC POTENTIAL}

Molecular imaging of the unique regions involved in post-MI LV remodeling holds great prognostic importance. Noninvasive, longitudinal assessment of qualities such as the fiber architecture and metabolism of the border zone hold the potential to risk-stratify patients. By identifying those at risk for continued post-MI adverse remodeling, we can direct these patients to adjuvant therapies in an effort to reduce the morbidity of the LV remodeling process. Hence molecular imaging techniques hold promise for further individualizing the treatments patients receive after MI.

Given the prevalence and dire consequences of the LV remodeling process, a multitude of devices and therapies have been developed in an effort to battle this clinical entity. The high-quality images produced by cardiac MRI have become an excellent tool for guiding the use of such technologies. One specific group of devices aimed at attenuating LV remodeling after MI are ventricular restraint devices, which apply epicardial pressure to combat dilation of the LV and reduce LV wall stress (35,36). Magovern et al. used cardiac MRI to evaluate the placement of a nitinol mesh device (Paracor Surgical Inc.) in an ovine MI model. MRI at 6 wk after MI demonstrated less increase in LV enddiastolic volume index and end-systolic volumes in devicetreated animals than in controls, as shown in Figure 3 (35). This study set the stage for future work that would use MRI techniques to characterize the regional effects of a cardiac restraint device on post-MI remodeling. Specifically, Blom et al. used cardiac MRI and novel 3-dimensional surface modeling techniques to measure the degree of infarct expansion and global LV remodeling in an ovine MI model with the CorCap (Acorn Cardiovascular) ventricular restraint device (36). The study found that animals treated with the device at $3 \mathrm{~d}$ after MI had a decreased infarct area and improved contractile function in the border zone, compared with control animals at 12 wk after MI (36). Device-treated animals demonstrated no expansion in either the infarcted or perfused regions after device placement, compared with control animals, which displayed more than $300 \%$ expansion in the surface area of the infarct region. These results supported the conclusion that early ventricular restraint after MI is effective in limiting infarct expansion and LV remodeling. Cardiac MRI techniques have transitioned the examination of these specific devices from preclinical studies to clinical application, providing valuable noninvasive follow-up capabilities (Fig. 4).
Another field that has drawn great attention in the treatment of LV remodeling is the use of cellular therapies. Specifically, the use of various types of stem cells in the postMI context has been extensively studied (37-39). MRI techniques using magnetically labeled stem cells have demonstrated the ability to track these cells after delivery to the myocardium. In a preclinical study, Kim et al. used MRI techniques to track magnetically labeled mesenchymal stem cells for up to $3 \mathrm{mo}$ after injection into a prior MI site and to assess the functional response to the cell injection (37). The study found that the cells were able to be seen on MRI up to $10 \mathrm{wk}$ after injection (Fig. 5) and that LV ejection fraction was improved, compared with control animals. Using similar MRI techniques, Hill et al. demonstrated the ability to track iron fluorophore particle-labeled mesenchymal stem cells in a porcine model up to $3 \mathrm{wk}$ after MI (Fig. 6). The study also demonstrated that the iron-labeled mesenchymal stem cells retained in vitro viability, proliferation, and differentiation
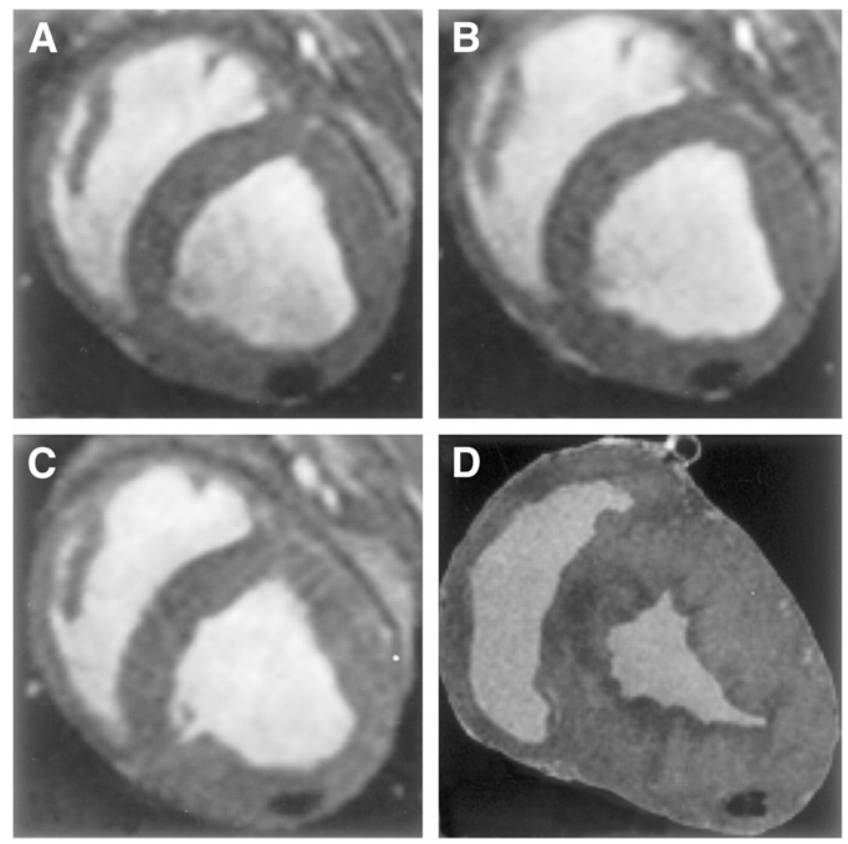

FIGURE 6. Serial in vivo and ex vivo MRI. Serial short-axis views of diastolic frames show persistent signal void after injection of $10^{5}$ mesenchymal stem cells labeled with iron fluorophore particles. Imaging was performed on days $1(A)$, 4 (B), and 21 (C). (D) Corresponding view of explanted heart on high-resolution 3-dimensional MRI showing signal void. (Reprinted with permission of (38).) 
capability as well as in vivo viability after allogeneic transplantation (38). The minimum detectable amount of cells on serial MRI was found to be $10^{5}$ cells per injection. These findings make MRI detection of iron-labeled cells an attractive alternative for the noninvasive evaluation of cellular therapies. This technique holds the potential to answer questions about cell engraftment, migration, and honing, to elucidate disparate results from early clinical trials involving cellular therapies in the post-MI context $(38,39)$.

\section{CONCLUSION}

The post-MI remodeling process involves a heterogeneous anatomic and physiologic regional LV landscape. Although this complex landscape is rife for therapeutic intervention, its intricacies must first be elucidated. Each unique anatomic region holds potential targets to attenuate the progression toward heart failure. With the assistance of advancing cardiac MRI techniques, these potential targets will progress toward becoming valuable clinical therapies, and current treatment modalities will continue to be refined.

\section{REFERENCES}

1. Krumholz HM, Anderson JL, Bachelder BL, et al. ACC/AHA 2008 performance measures for adults with ST-elevation and non-ST-elevation myocardial infarction: a report of the American College of Cardiology/American Heart Association Task Force on Performance Measures (Writing Committee to Develop Performance Measures for ST-Elevation and Non-ST-Elevation Myocardial Infarction). J Am Coll Cardiol. 2008;52:2046-2099.

2. Russo CA, Andrews RM. The National Hospital Bill: The Most Expensive Conditions, by Payer, 2004. Rockville, MD: Agency for Healthcare Research and Quality; 2006. HCUP statistical brief no. 13.

3. Cleutjens JP, Kandala JC, Guarda E, Guntaka RV, Weber KT. Regulation of collagen degradation in the rat myocardium after infarction. J Mol Cell Cardiol. 1995;27:1281-1292.

4. Judd JT, Wexler BC. Prolyl hydroxylase and collagen metabolism after experimental myocardial infarction. Am J Physiol. 1975;228:212-216.

5. White HD, Norris RM, Brown MA, Brandt PW, Whitlock RM, Wild CJ. Left ventricular end-systolic volume as the major determinant of survival after recovery from myocardial infarction. Circulation. 1987;76:44-51.

6. McCormick RJ, Musch TI, Bergman BC, Thomas DP. Regional differences in LV collagen accumulation and mature cross-linking after myocardial infarction in rats. Am J Physio. 1994;266:H354-H359.

7. Jugdutt BI, Lucas A, Khan MI. Effect of angiotensin-converting enzyme inhibition on infarct collagen deposition and remodelling during healing after transmural canine myocardial infarction. Can J Cardiol. 1997;13:657-668.

8. Jugdutt BI, Joljart MJ, Khan MI. Rate of collagen deposition during healing and ventricular remodeling after myocardial infarction in rat and dog models. Circulation. 1996;94:94-101.

9. van den Borne SW, Isobe S, Verjans JW, et al. Molecular imaging of interstitial alterations in remodeling myocardium after myocardial infarction. $J$ Am Coll Cardiol. 2008;52:2017-2028.

10. Harada K, Friedman M, Lopez JJ, et al. Vascular endothelial growth factor administration in chronic myocardial ischemia. Am J Physiol. 1996;270:H1791H1802.

11. Voisine P, Bianchi C, Ruel M, et al. Inhibition of the cardiac angiogenic response to exogenous vascular endothelial growth factor. Surgery. 2004;136:407-415.

12. Boodhwani M, Sodha NR, Mieno S, et al. Functional, cellular, and molecular characterization of the angiogenic response to chronic myocardial ischemia in diabetes. Circulation. 2007;116(11, suppl)I31-I37.

13. Meoli DF, Sadeghi MM, Krassilnikova S, et al. Noninvasive imaging of myocardial angiogenesis following experimental myocardial infarction. J Clin Invest. 2004;113:1684-1691.

14. Wilson EM, Moainie SL, Baskin JM, et al. Region- and type-specific induction of matrix metalloproteinases in post-myocardial infarction remodeling. Circulation. 2003;107:2857-2863.
15. Dixon JA, Gorman RC, Stroud RE, et al. Mesenchymal cell transplantation and myocardial remodeling after myocardial infarction. Circulation. 2009;120(11, suppl):S220-S229.

16. Ekhterae D, Hinmon R, Matsuzaki K, et al. Infarction induced myocardial apoptosis and ARC activation. J Surg Res. June 6, 2009 [Epub ahead of print].

17. Hamamoto H, Gorman JH III, Ryan LP, et al. Allogeneic mesenchymal precursor cell therapy to limit remodeling after myocardial infarction: the effect of cell dosage. Ann Thorac Surg. 2009;87:794-801.

18. Jugdutt BI, Menon V, Kumar D, Idikio H. Vascular remodeling during healing after myocardial infarction in the dog model: effects of reperfusion, amlodipine and enalapril. J Am Coll Cardiol. 2002;39:1538-1545.

19. Sosnovik DE, Nahrendorf M, Panizzi P, et al. Molecular MRI detects low levels of cardiomyocyte apoptosis in a transgenic model of chronic heart failure. Circ Cardiovasc Imaging. 2009;2:468-475.

20. Grothues F, Smith GC, Moon JC, et al. Comparison of interstudy reproducibility of cardiovascular magnetic resonance with two-dimensional echocardiography in normal subjects and in patients with heart failure or left ventricular hypertrophy. Am J Cardiol. 2002;90:29-34.

21. Azevedo CF, Cheng S, Lima JA. Cardiac imaging to identify patients at risk for developing heart failure after myocardial infarction. Curr Heart Fail Rep. 2005;2:183-188.

22. Friedrich MG, Abdel-Aty H, Taylor A, Schulz-Menger J, Messroghli D, Dietz R. The salvaged area at risk in reperfused acute myocardial infarction as visualized by cardiovascular magnetic resonance. J Am Coll Cardiol. 2008;51:1581-1587.

23. Weis S, Shintani S, Weber A, et al. Src blockade stabilizes a Flk/cadherin complex, reducing edema and tissue injury following myocardial infarction. J Clin Invest. 2004;113:885-894.

24. Lima JA, Judd RM, Bazille A, Schulman SP, Atalar E, Zerhouni EA. Regional heterogeneity of human myocardial infarcts demonstrated by contrast-enhanced MRI: potential mechanisms. Circulation. 1995;92:1117-1125.

25. Ito H, Maruyama A, Iwakura K, et al. Clinical implications of the "no reflow" phenomenon: a predictor of complications and left ventricular remodeling in reperfused anterior wall myocardial infarction. Circulation. 1996;93:223-228.

26. Wu KC, Zerhouni EA, Judd RM, et al. Prognostic significance of microvascular obstruction by magnetic resonance imaging in patients with acute myocardial infarction. Circulation. 1998;97:765-772.

27. Beek AM, Kühl HP, Bondarenko O, et al. Delayed contrast-enhanced magnetic resonance imaging for the prediction of regional functional improvement after acute myocardial infarction. J Am Coll Cardiol. 2003;42:895-901.

28. Maes A, Van de Werf F, Nuyts J, Bormans G, Desmet W, Mortelmans L. Impaired myocardial tissue perfusion early after successful thrombolysis: impact on myocardial flow, metabolism, and function at late follow-up. Circulation. 1995;92:2072-2078.

29. Gerber BL, Belge B, Legros GJ, et al. Characterization of acute and chronic myocardial infarcts by multidetector computed tomography: comparison with contrast-enhanced magnetic resonance. Circulation. 2006;113:823-833.

30. Nieman K, Shapiro MD, Ferencik M, et al. Reperfused myocardial infarction: contrastenhanced 64-section CT in comparison to MR imaging. Radiology. 2008;247:49-56.

31. Wu EX, Wu Y, Nicholls JM, et al. MR diffusion tensor imaging study of postinfarct myocardium structural remodeling in a porcine model. Magn Reson Med. 2007;58:687-695.

32. Wu MT, Tseng WY, Su MY, et al. Diffusion tensor magnetic resonance imaging mapping the fiber architecture remodeling in human myocardium after infarction: correlation with viability and wall motion. Circulation. 2006;114:1036-1045.

33. Hu Q, Wang X, Lee J, et al. Profound bioenergetic abnormalities in peri-infarct myocardial regions. Am J Physiol Heart Circ Physiol. 2006;291:H648-H657.

34. Feygin J, Mansoor A, Eckman P, Swingen C, Zhang J. Functional and bioenergetic modulations in the infarct border zone following autologous mesenchymal stem cell transplantation. Am J Physiol Heart Circ Physiol. 2007;293:H1772-H1780.

35. Magovern JA, Teekell-Taylor L, Mankad S, et al. Effect of a flexible ventricular restraint device on cardiac remodeling after acute myocardial infarction. ASAIO J. 2006;52:196-200.

36. Blom AS, Pilla JJ, Arkles J, et al. Ventricular restraint prevents infarct expansion and improves borderzone function after myocardial infarction: a study using magnetic resonance imaging, three-dimensional surface modeling, and myocardial tagging. Ann Thorac Surg. 2007;84:2004-2010.

37. Kim YJ, Huh YM, Choe KO, et al. In vivo magnetic resonance imaging of injected mesenchymal stem cells in rat myocardial infarction: simultaneous cell tracking and left ventricular function measurement. Int J Cardiovasc Imaging. 2009;25(suppl 1):99-109.

38. Hill JM, Dick AJ, Raman VK, et al. Serial cardiac magnetic resonance imaging of injected mesenchymal stem cells. Circulation. 2003;108:1009-1014.

39. Kraitchman DL, Heldman AW, Atalar E, et al. In vivo magnetic resonance imaging of mesenchymal stem cells in myocardial infarction. Circulation. 2003;107:2290-2293. 